\title{
The Role of Norepinephrine in Amygdala Dependent Fear Learning and Memory
}

\author{
Sodikdjon A. Kodirov
}

Additional information is available at the end of the chapter

http://dx.doi.org/10.5772/51448

\section{Introduction}

"The amygdaloid complex in the rat is very pronounced"

Gurdjian, 1928

The extensive knowledge about the significance and connexions of the amygdala with other brain regions emerged in the early XXth century (Gurdjian, 1928). Since then, the seven comprising nuclei of amygdala - corpus amygdaloideum in rats, have been known (Brodal 1947; Cowan et al., 1965). The presence and stereotaxic locations of all nuclei were confirmed later with more contemporary approaches (Paxinos \& Franklin, 2001; Mikula et al., 2007). The additional nuclei belonging to the amygdala are established as "extended amygdala" (Fig. 1) that includes also the bed nucleus of the stria terminalis (BNST). However, the majority of studies address the lateral (LA), basolateral (BLA) and central (CeA) amygadala and their role in several emotionally driven responses in organisms. One of the latter responses is the stress and its circuit initiates in locus coeruleus (LC) after a release of norepinephrine (NE) by noradrenergic neurons.

Similar to other catecholamines such as epinephrine (adrenaline) and dopamine (DA), norepinephrine (noradrenaline) is released either into the circulation or locally to brain regions as a response to stress. NE subsequently also is transmitted into the amygdala. An increase in NE content within the amygdala modulates multiple physiological functions. In the amygdala the content of NE is higher than DA, but comparable to that of serotonin (Niwa et al., 2011). The noradrenergic neurons are present in the LC and amygdala. These neurons are distinguished by their positive reaction to $\mathrm{DBH}$ - dopamine $\beta$-hydroxylase, which enables the conversion of DA into NE. The physiological (endogenous) dynamics or pathological increase of NE occur at terminals originating from the LC (Emson et al., 1979). In individuals with a history of post-traumatic stress disorder (PTSD) the properties of the amygdala, along the hippocampus and prefrontal cortex, are affected and involve stress 
hormones such as cortisol and NE (for review see Bremner, 2006). Specifically, the fearconditioning paradigm bilaterally increases the activity of the amygdala in those with symptoms of PTSD as a result of abuse. In healthy subjects, such an increase targets the left hemisphere; nevertheless, the left amygdala in the PTSD group is more active compared to the control. The upregulated activity in the amygdala correlates with increased blood flow to this region in the PTSD group.

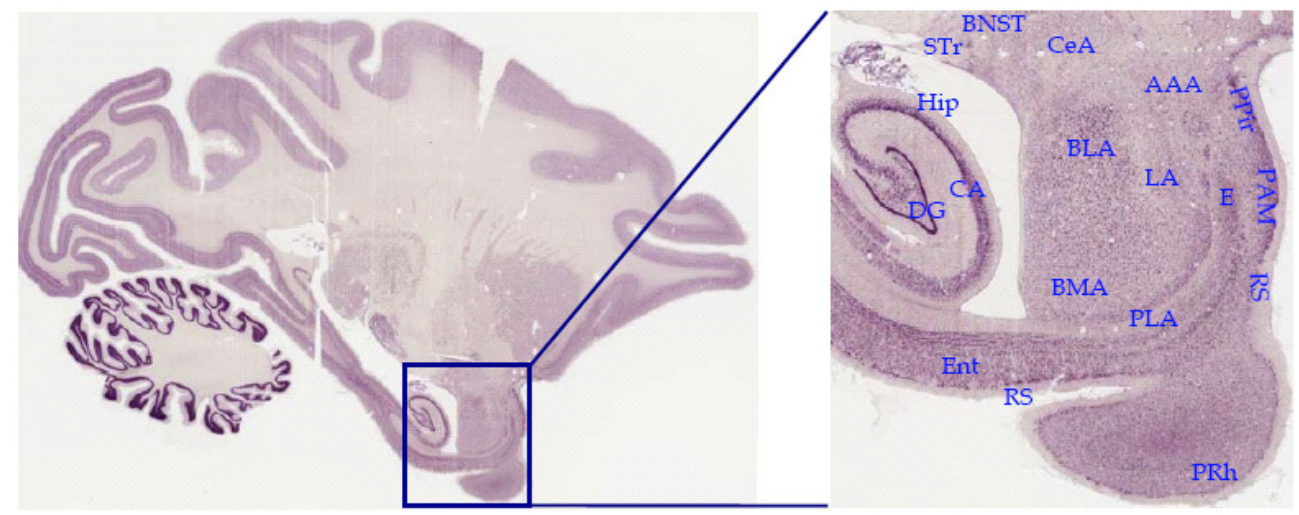

Figure 1. Amygdala of rhesus monkey Macaca mulatta

Left, whole brain in sagittal plane and right, amygdala and adjacent regions. AAA - anterior amygdaloid area, BLA - basolateral amygdala, BMA - basomedial amygdala, BNST - bed nucleus of the stria terminalis, CA - cornu ammonis, CeA - central amygdaloidal nucleus, Ent - entorhinal cortex, DG - dentate gyrus, Hip - hippocampus, LA - lateral amygdala, PRh - perirhinal cortex, PLA paralaminar nucleus of amygdala, PMA - periamygdaloid area, PPir - prepiriform cortex, RS - rhinal sulcus, STr - stria terminalis (www.brainmaps.org and Mikula et al., 2007).

\section{Emotional learning and resultant memories via amygdala}

The amygdala is the main brain region responsible for emotion, at least for its intensity. Emotions, in turn, can influence memory leading to either forgetting - amnesia or stronger (compared to average) remembering - hypermnesia. The latter two events occur in anterograde or retrograde fashion.

The emotional fear response originates in the brain by the convergence (Fig. 2) of conditioned and unconditioned stimuli (CS and US), the latter can be also shown in experimental animals by employing diverse parameters depending on the area of interest (Halverson et al., 2009; Kwapis et al., 2009). A particular Pavlovian training or conditioning that has been widely used in research, enables experimental animals to associate the neutral CS with the US of a negative valence. This association results in an aversive response to the subsequently encountered CS. Fear conditioning paradigms are excellent tools for the study of neurobiological substrates of learning and memory. With regard to this, a peculiar interest has been devoted to the role of amygdala in auditory fear conditioning (LeDoux et al., 1984). The learning process in the amygdala and resultant memory undergo multiple 
steps involving consolidation and re-consolidation. Although the mechanisms underlying these two steps to a greater extent are controversial (Alberini, 2008). The interplay between information storage and dynamic properties of synapses are complex (Varshney et al., 2006). The consolidation of recent memories (from the previous day) are processed during sleep within the rapid eye movement (REM) phase, the latter leads to sorting of relatively earlier (then the very recent one) acquired information and its subsequent forgetting (Poe et al., 2000). Fear memory and circuits related to addiction often function synergistically (Peters et al., 2009).

The pairing paradigm that is equivalent to Pavlovian (light-food pairings) classical conditioning (Pavlov, 1927) enables the potentiated response of synapses in the amygdala also in vitro (Nader et al., 2000; McKernan \& Shinnick-Gallagher, 1997; Rogan et al., 1997). Fear conditioning and the strength of acquired memory parallel the increased numbers of reactivated neurons (Reijmers et al., 2007) both in the LA and BLA. However, we should consider that not only the amygdala, but also the hippocampus participates in fear response and its subsequent processing (Knierim, 2003). The modulation of cognition by NE is also continuously elucidated in human subjects concurrently with established paradigm.

The neuronal excitability is a result of activities and properties of multiple ion channels including hyperpolarization-activated cyclic nucleotide gated non-selective cation (HCN or Ih) channels. It has been established that NE possesses the inverted U shaped dose response effects and targets the $\alpha 2 \mathrm{~A}$ adrenergic receptors (AR) and inhibits HCN channels (for review see Arnsten, 2007). Postsynaptic action potentials and neuronal excitability are required for the plasticity within several inputs. The neuronal excitability in turn is also prone to plasticity that indirectly involves the HCN channels (Brager \& Johnston, 2007).

\subsection{Clinical experiments}

Cortisol and NE are considered as a main stress hormones (for literature analyses see Cahill et al., 2003). The authors reported that cortisol levels in saliva samples increases from $\sim 3.5$ to $4.2 \mathrm{ng} / \mathrm{ml}$ in response to a simple paradigm - cold pressor stress (CPS). In those subjects (control) who immersed the left forearm up to above the elbow joint level into the slightly warmer water $\left(37-40^{\circ} \mathrm{C}\right)$ than body temperature, the concentration of cortisol decreased to $\sim 3 \mathrm{ng} / \mathrm{ml}$ when compared to CPS group $\left(0-3{ }^{\circ} \mathrm{C}\right)$. Under these two conditions, subjects exhibited similar LTM for neutral pictures. Greater amounts of emotionally charged pictures were correctly recalled by CPS group.

Stressful events result in activation of the amygdala accompanied by increase in NE levels and surgery counts to this. Patients who underwent general anesthesia responded faster and provided correct word associations with emotionally negative cues compared to neutral ones (Gidron et al., 2002). This was the case when it concerned the old cues, however in regard to new cues the opposite effects were observed. Moreover, there was a correlation between the reaction time and spectral edge frequency (SEF) during EEG recordings. Thus, analyses revealed that patients with a SEF of lower than $9 \mathrm{~Hz}$ reacted slower. 
It has been shown that the variation in NE levels can mimic the intensity of emotion in tested subjects (Hurlemann et al., 2005). In this study, the noradrenergic response to NE was inhibited by propranolol via blockade of $\beta$-adrenoceptors. Experimentally, the noradrenergic response was enhanced by NE reuptake inhibitor, reboxetine mesilate, a pharmaceutical that is widely used in order to reveal the resultant changes during the exposure to certain experimental paradigms. Former treatment decreased the arousal to oddball stimuli, while the latter increased it. Such outcomes were observed with both positive and negative oddball stimuli, but not with neutral ones. Moreover, the valence during all three paradigms remained unchanged. Propranolol, but not reboxetine, lowered the systolic and diastolic blood pressure. The plasma levels of these drugs at the end of recall paradigm were 20 and $75 \mu \mathrm{g} / \mathrm{L}$, respectively. In fact, the plasma content depends on overall body's metabolism and prior fasting, as was shown for reboxetine (Hurlemann et al., 2007). Although, emotionally driven retrograde amnesia can occur via modulation of either NE or cortisol signaling, concurrent activation of these systems is perhaps an adequate underlying mechanism. This notion is supported by the magnitudes of recall change under negative emotion contact (E-1) for reboxetine (34\%) and synthetic cortisol $(24 \%)$, hydrocortisone, alone. The latter was increased by co-application of both (43\%). The amnesic influence was seen also on adjacent E-2 contact (22\%) and lasted $10 \mathrm{~s}$. All these treatment combinations did not alter the von Restroff phenomenon, and the correct recall magnitudes were $\sim 95 \%$ for oddball stimuli.

Oral intake of yohimbine reversibly increases the content of $\alpha$-amylase in human saliva (van Stegeren et al., 2010). A cortisol containing pill enhanced the endogenous cortisol level in these subjects abruptly and sustained at plateau for at least $1 \mathrm{~h}$. The baseline content was documented after one week. The performance of these two groups, in terms of (better) recognition and recalling emotional pictures, when compared to neutral ones were similar. Yohimbine was ineffective while cortisol improved recognition and recall responses to both stimuli to similar extent. The combination of both agents strengthened the response to the emotional stimulus. Endogenous cortisol levels in human subjects vary greatly, and for this reason, experiments are often conducted by assigning two groups with relatively low ( $\sim 5$ $\mathrm{nM})$ and high ( $\sim 8 \mathrm{nM})$ contents (van Stegeren et al., 2007). Under placebo the magnitude of amygdalar activation in response to emotional pictures (compared to those of neutral nature) correlated well with the level of cortisol within the groups. It was suggested that the response of the amygdala underlies an increase in NE levels, since after the intake of $80 \mathrm{mg}$ propranolol such correlations were absent. Additionally, propranolol increased the cortisol levels, but not to significant extent.

The cortisol concentration changes dynamically, with the highest level ( $12 \mu \mathrm{g} / \mathrm{dl})$ occurring during the late (REM) sleep. Oral intake of $3 \mathrm{~g}$ metyrapone (cortisol synthesis inhibitor) before the sleep inhibited the plasma level of cortisol during $8 \mathrm{~h}$ of sleep in male subjects (Wagner et al., 2005). Metyrapone increased the plasma content of NE during both learning (from 100 to 120) and retrieval ( 130 vs. $160 \mathrm{pg} / \mathrm{ml}$ ) and its concentration was lower during sleep. These opposing effects of metyrapone on cortisol and NE dynamics correlate and promote the emotionally charged memory formation that occurs within the amygdala. 
Emotional memories are consolidated during the slow wave sleep (SWS) and involve NE release. This was verified by intravenous infusion of $\alpha 2$ agonist clonidine, which inhibits NE release by the LC and decreases the retention of temporal order of emotional stories (Groch et al., 2011). Clonidine inhibited only the REM phase, which consisted of about $5 \%$ of total sleep time in tested subjects.

The NE degradation occurs by catechol-O-methyltransferase (COMT). In case of polymorphism in the COMT gene, the substitution of valine by methionine occurs at amino acid 158 (val ${ }^{158}$ met). In healthy subjects, the particular allele of the COMT gene is associated with emotional memory formation in the amygdala (Smolka et al., 2005). The unpleasant stimulus (pictures) was found to activate the right human amygdala as revealed by blood oxygen level-dependent (BOLD) response during fMRI scanning. The highest activity was observed in met ${ }^{158}$ homozygous individuals compared to val ${ }^{158}$ homozygous or val/met heterozygous.

Van Stegeren et al. (2005) confirmed that NE is a neurotransmitter involved in memories of emotional nature. Propranalol slightly increased the baseline heart rate (HR) compared to placebo group, but it significantly decreased before and after the fMRI procedure. Note that the procedure itself lowered the HR in both groups. Both groups similarly distinguished the emotional intensity of presented pictures by comparing them to prior images. The responses to gradually increased emotional intensity of pictures correlated with the pattern of activity in the amygdala. The latter activity was decreased by propranolol, but to significant extent only at intermediate intensity. In females, the amygdala exhibited about two fold less activation and higher resistance to propranolol compared to males. Male and female subjects also rated the pictures of similar intensity differently, especially former identified a greater number of images of neutral nature, while the latter rated them as emotionally intense pictures. Interestingly, propranolol did not affect the response of subjects exposed to pictures of highest emotional intensity. The overall memory performance was similar in both genders (van Stegeren et al., 2005).

\subsection{Animal models}

\subsubsection{In vivo studies - behavior}

The memory performance and related behaviour in rodents can be analyzed by employing multiple trainings and tests. The existing findings reflect controversial roles for NE in amygdala dependent memory.

\subsubsection{Studies revealing the enhancement of memory by $N E$}

Since moderate stress promotes memory formation and this event is accompanied by the release of NE, it is logical to expect similar effects on retention by this substance alone. The retention of memory is often manifested during the object recognition task. The overall performance depends on the duration of training, i.e. rats exploring objects for 3 min can retain memories for only one hour. By increasing the training time to $10 \mathrm{~min}$, one would observe the resultant retention even after $24 \mathrm{~h}$ (Roozendaal et al., 2008). The latter study 
revealed that the NE administration into the BLA immediately after 3 min training improved the retention that lasted at least $24 \mathrm{~h}$. However, the NE was effective only at lower doses up to $1 \mu \mathrm{g}$ and declined abruptly at $3 \mu \mathrm{g}$. Direct exposure of BLA to propranolol resulted in impairment of retention in those rats trained during $10 \mathrm{~min}$. The enhancement of fear memory by bilateral injection of NE into the BLA is reversed by prior exposure to context (Huff et al., 2005). In this regard, the latter study also provides some clarifications addressing controversial arguments in several studies. The dose response was not classical as judged by three different concentration of NE, and a clear effect was evident only at $1 \mu \mathrm{g}$, since at $3 \mu \mathrm{g}$, the freezing response - memory for fear, among tested rats declined and was not significant compared to control group. Injection of propranolol into the BLA immediately after training abolishes the enhancement of object recognition memory by corticosterone (Roozendaal et al., 2006b). The blockade of this memory via $\beta$-adrenoceptors was selective to the amygdala, since similar procedures (albeit even higher concentration of antagonist) targeting the hippocampus did not alter the discrimination index.

There are also toxins, which target noradrenergic neurons. One of them is DSP-4 [N-(2chloroethyl)-N-ethyl-2 bromobenzylamine] with selective effects on DBH positive cells of the amygdala and LC as demonstrated 10 days after i.p. injections in adult rats (Radwanska et al., 2010). The toxicity effect on BLA neurons was more pronounced compared to LC ones. One week after the injection, animals underwent the habituation and training sessions, and active avoidance responses to US were analyzed. The majority of DSP-4 treated rats were unable to avoid the foot-shock within the $5 \mathrm{~s}$ consequently receiving $25 \mathrm{~s}$ long US. The same tendency was found also after additional seven training sessions in subsequent days and correlated with the decrease in NE neurons. The DBH positive neurons in BLA are also immunoreactive to choline acetyltransferase (ChAT). Moreover, DBH and ChAT positive terminals can also be found in close proximity, perhaps even are synaptically connected to the same neuron (Li et al., 2001).

Long lasting increase in NE level were observed in response to $0.55 \mathrm{~mA}$ foot-shock applied during $1 \mathrm{~s}$ via the floor in dark compartment of the inhibitory avoidance box (Mcintyre et al., 2002). The immediate mean release after the shock was estimated around two-fold compared to baseline. The three-fold peak increase in NE level occurred after $15 \mathrm{~min}$ and then gradually declined, but did not reach baseline during up to $2 \mathrm{~h}$ and remained at $\sim 1.5$ fold. Interestingly, the identical stimulus delivered via the grid in the bottom of the holding cage evoked a small NE release that lasted $\sim 15 \mathrm{~min}$. In some rats, the content of NE reached the highest level ( 7.5 fold), and there was to some extent a correlation between the level of $\mathrm{NE}$ and the latency to enter the dark compartment during inhibitory avoidance (IA) test in particular animals. Rats injected i.p. with corticosterone immediately after IA training retain memories 10 fold longer ( $\sim 300$ vs $\sim 30$ s) compared to controls (McReynolds et al., 2010). Interestingly, this procedure resulted in a transient two-fold increase in NE level in BLA and Arc expression in the hippocampus; the latter effects were observed only in trained rats. In fractioned synaptoneurosome, the expression of PSD-95 was higher than in total homogenate from hippocampus. Direct injection of propranolol into the BLA decreased the Arc density in synaptoneurosome preparation. These results support the notion that 
amygdala and hippocampus may act in synergy during cognitive behavior. Note that the increase in NE levels of similar magnitude ( 1.6 fold) should occur even in the absence of any drug in the amygdala after IA training, and cAMP response element-binding (CREB) antisense reduces its magnitude and duration (Canal et al., 2008). The clenbuterol administration immediately following the training improved the related memory.

One of earlier studies demonstrated that the systemic injection of epinephrine increases the NE release in the amygdaloid complex in a reversible manner (Williams et al., 1998). Its magnitude was comparable when either 0.1 or $0.3 \mathrm{mg} / \mathrm{kg}$ epinephrine were used. The authors compared these effects with those resulting after escapable foot-shock in these two groups. The $1 \mathrm{~s}$ foot-shock with the intensity of $0.8 \mathrm{~mA}$ caused only a slight NE increase in of groups, but differences appeared not to be significant. The NE increase was concluded to take place in amygdalar terminals of nucleus tractus solitarius (NTS) neurons. The latter was confirmed recently, since experimentally NE release could be also achieved in BNST extended amygdala - by stimulating $(60 \mathrm{~Hz})$ the fibers of NTS. Such stimulation evokes higher NE release in rats intraperitoneally injected with either idazoxan (selective $\alpha 2$ adrenergic receptor antagonist) or desipramine (NE reuptake inhibitor). The evoked release is distinctly modulated by averse and pleasant stimuli (Park et al., 2011), thereby it is either increased or decreased in response to intra-oral delivery of quinine and sucrose (palatable food). Both substances affected the magnitude of NE release into the BNST to similar extent $( \pm 20 \mathrm{nM})$ with the time course of around 9 s. The content of NE metabolite, MHPG (3methoxy-4-hydroxyphenylglycol), also changes in those areas of brain that possess noradrenergic terminals. The ratio of MHPG to NE is significantly increased within BNST when animals are fear conditioned (Onaka \& Yagi, 1998).

The post-training injection of NE into the BLA enhanced the retention of contextual fear conditioning (CFC) revealed by freezing time during the Y-maze test (LaLumiere et al., 2003). The pattern of response to two different concentrations was similar when the latency of both freezing and entry into the shock arm were analyzed. The CFC could be performed also by using the straight alley test and analyzing the avoidance (latency) of rats to enter the dark shock compartment. The enhancement of retention by $1 \mu \mathrm{g}$ NE was comparable to that in the Y-maze, but slightly less pronounced ( 3 vs. 4 fold).

Finally, the improvement of the retention is also observed during antagonism of $\alpha 2$ adrenoceptors by idazoxan (Ferry \& McGaugh, 2008). The effects of idazoxan differ depending on either pre- or post IA training injections. However, in both cases this selective $\alpha 2$ antagonist improves retention. Thus, idazoxan injected into the BLA $20 \mathrm{~min}$ prior to IA test increased the retention latency from $\sim 120$ to $180 \mathrm{~s}$, which was significantly longer ( 260 s) when introduced immediately after foot-shock. The dose-dependent effects of idazoxan in these two experiments were similar and the peak identically occurred at $0.3 \mu \mathrm{g}$ demonstrating the narrow bell-shaped effects. In another group, increasing the foot-shock intensity from 0.4 to $0.5 \mathrm{~mA}$ resulted in prolonged retention latency to $\sim 300 \mathrm{~s}$ and that was decreased by injection of agonist UK 14,304 (up to $3 \mathrm{ng}$ ). The subcutaneous injection of hormone corticosterone decreased the conditioned auditory-cue fear response (Roozendall et al., 2006a). The effect of corticosterone was reversed by injection of $\beta 1$-adrenoceptor 
antagonist atenolol $(0.5 \mu \mathrm{g})$ into the BLA. Atenolol alone was ineffective and both agents' effects occur only when they were administered immediately after pairing of tone with the shock, but not before it.

\subsubsection{Studies revealing the impairment of memory by NE}

Most evidently, severe stress negatively impacts the memory and correlated amount of NE released during this period may exert such effects. A dramatic release of multiple neurotransmitters including NE $(1200 \%)$ is observed also after bilateral injection of antibiotic anisomycin into the amygdala. However, the latter led to amnesia (Canal et al., 2007). Interestingly, the vehicle injection increased NE levels by $\sim 200 \%$. Under both conditions, the NE release was a transient event lasting $\sim 60$ and $45 \mathrm{~min}$ in former and latter cases. Another difference was seen in samples from anisomycin treated group that showed a rebound decrease almost to $0 \%$ below the normalized $100 \%$ baseline level. The baselines were not strictly stable, but to some extent identical in both groups. A rebound decrease was not observed in vehicle-injected animals, and after a transient increase, the NE levels returned to baseline values. When samples were analyzed every $45 \mathrm{~min}$ (vs. $15 \mathrm{~min}$ above), the transient increase was less pronounced in the anisomycin group, while it disappeared in the vehicle one. The rebound decrease in NE release was consistently present in anisomycin treated animals and remained at $50 \%$ despite the prolonged experiments; baseline recovery occurred after $48 \mathrm{~h}$. Amnesia by anisomycin involves the noradrenergic receptors, since either the prior injection of propranolol or subsequent administration of clenbuterol ( $\beta 2$ agonist) resulted in significantly lower impairment of memory in both groups of experiments. The lidocaine $\left(\mathrm{Na}^{+}\right.$channel blocker) prevents $\mathrm{NE}$ release that is evoked by intra-amygdalar injection of anisomycin (Sadowski et al., 2011). The latter correlated with the reversal (to some extent) of memory impairment achieved by anisomycin. Furthermore, anisomycin attenuated c-Fos (cellular FBJ osteosarcoma oncogene) immunoreactivity (assessed by application of foot-shocks) by inhibiting the protein synthesis in BLA, and thereby providing some possible challenges for required de novo synthesis for long-term memory generation. The activity of c-Fos within several major nuclei of amygdala was up to 10 fold higher after $2 \mathrm{~h}$ of contextual fear training (Murchison et al., 2011). This increase was independent of the presence of ligand for adrenergic receptors (NE and E) as in the case of previously established $\mathrm{Dbh}^{--}$mice (see Murchison et al., 2011). Under these conditions, the lowest level of c-Fos was in the CeA. However, upon reexposure to context $1 \mathrm{~d}$ after conditioning the immunoreactivity was highest among tested nuclei including the LA. Moreover, in $\mathrm{Dbh}^{-/}$mice both the c-Fos level and magnitude of freezing (after contextual fear conditioning) were decreased compared to $\mathrm{Dbh}^{+-}$as a result of impaired memory retrieval. No differences were seen when mice were introduced to context at seven days or after that. Since in $\mathrm{Dbh}^{-/}$the complete adrenergic system is disrupted, the authors used also $\beta_{1}$ adrenergic receptor knockout $\left(\beta_{1} \mathrm{KO}\right)$ mice and obtained similar results.

Stress can be introduced to laboratory animals with paradigms closely resembling those occurring in the nature: social isolation, maternal separation or both. The combination of latter factors in mice between postnatal day 15 (P15) and P21 reduced the social interaction time by two-fold, measured during adulthood (Niwa et al., 2011). Thus, these mice were 
more anxious and show a decreased short-term memory (STM) compared to the control group, which is improved with antipsychotic drug clozapine. Under these conditions, the plasma level of corticosterone increased by four-fold (from $\sim 200$ to $\sim 800 \mathrm{pg} / \mathrm{ml}$ ). The NE content shows a tendency to decrease, but statistical analyses perhaps did not reveal a degree of significance contrary to that of the frontal cortex. Note that the overall level of NE in the frontal cortex was higher compared to the amygdala. Nevertheless, injection of NE into the right BLA immediately after the CFC decreases the freezing behavior in a dosedependent manner (Berlau and McGaugh, 2006). However, this U-shaped response was narrow as judged by three logarithmic concentrations $(0.3-3 \mu \mathrm{g})$ and show peak effects at 1 $\mu \mathrm{g}$.

The pain perception was found to be lower in two lines of transgenic mice that lack $\alpha 2 \mathrm{~A}$ adrenergic receptors (Davies et al., 2004). One of the agonists of these receptors odexmedetomidine (i.p.) decreased the flinch threshold from 0.17 to $0.16 \mathrm{~mA}$ in WT mice. The odexmedetomidine in $\alpha 2 \mathrm{~A}$ AR mice did not affect the flinch response, while in D97N mice it increased the threshold ( 0.18 vs. $0.20 \mathrm{~mA})$ that was not considered as an effect. In WT mice the freezing response was decreased, when odexmedetomidine was injected $30 \mathrm{~min}$ prior to fear conditioning, but not immediately after. This procedure also reduced the number of both P-CREB (phosphorylated) and c-Fos positive neurons in all three major nuclei of amygdala: LA, BLA and CeA. While the number of these two groups of neurons were comparable in LA and CeA, in BLA the content of P-CREB was five-fold and those of c-Fos two-fold.

\subsubsection{In vivo studies - electrophysiology}

Electrophysiological studies in vivo provided interesting results on brain structures and neurobiological processes underlying learning and memory. It was shown that amygdala modulates the LTP in dentate gyrus (DG) and the effects are derived selectively by LA and BLA nuclei, but not CeA (Akirav \& Richter-Levin, 2002). Moreover, the effects of ipsilateral (same hemisphere) activation of two former nuclei on LTP in DG depend on timing between the two stimulations sites. The stimulation of the BLA just $30 \mathrm{~s}$ prior to the perforant path (PP) activation enhances LTP, while $2 \mathrm{~h}$ intervals decrease it. Interestingly, both effects, i.e. the enhancement and inhibition occur via two stress hormones, since the NE and corticosterone depletions by DSP-4 and metyrapone respectively reversed it. Although, also the contralateral (opposite hemisphere) priming of BLA exhibits similar effects, it was not derived by $\mathrm{NE}$ and corticosterone release. Note that the electrical stimulation of BLA decreases the NE release in DG (Almaguer-Melian et al., 2005).

The above-mentioned paradigm is known as DG LTP reinforcement by BLA. The injection of $\sim 7 \mathrm{nM}$ propranolol into the DG five minute prior to the BLA reinforcing paradigm decreases the LTP in DG achieved by PP stimulation (Bergado et al., 2007). The magnitudes of initial potentiation caused by PP stimulation in both groups were almost identical. Note that the control group comprises data obtained after the $\mathrm{NaCl}$ injections into the BLA, LC and medial septum and figure legend states that propranolol was delivered into the BLA. 


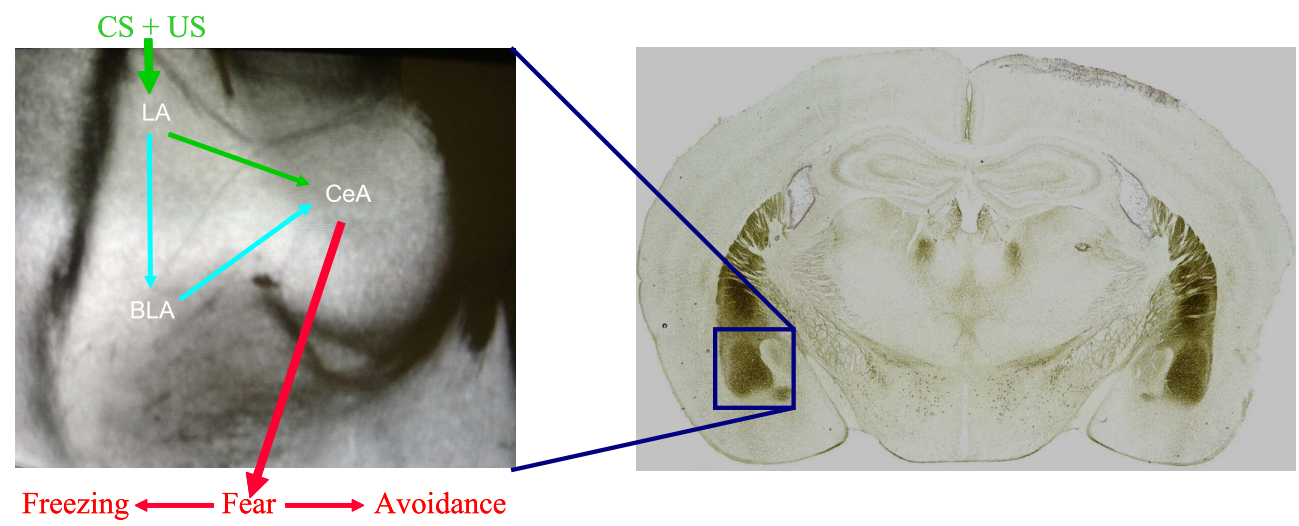

Figure 2. Amygdala and fear response pathways Left, amygdala (in actual coronal brain slice) and its major nuclei. Right, closely matching section of brain in coronal plane (Paxinos \& Franklin, 2001). LA - lateral amygdala, BLA - basolateral amygdala, $\mathrm{CeA}$ - central amygdaloidal nucleus. Schematic representation of fear response that originates after the convergence of conditioned and unconditioned stimuli (CS and US) into the LA.

\subsubsection{In vitro studies - cellular counterpart of learning and memory in rodents}

The potentiation of synaptic responses underlies the learning and memory at neuronal level.

\subsubsection{Characteristics of $L A$ and BLA cells involved in plasticity}

Diverse cells have been observed in the amygdala, but the majority is comprised of two types. They are pyramidal and interneurons, and both are prone to plasticity (Mahanty \& Sah, 1998; Tully et al., 2007). These neurons are distinguished by means of various methods; however, their electrophysiological properties could be considered a main criterion (Kodirov et al., 2009). In recent years, the attempt to recognize both types of cells by usage of biological marker - green fluorescent protein (GFP) - are starting to emerge. The interneurons in the BLA are apparently similar to other brain regions, because they express glutamate decarboxylase - 67 (GAD-67). In the BLA a single glutamatergic and three distinct GABAergic types of neurons have been distinguished (Kaneko et al., 2008). Although, this study is substantial, the characterized cell types warrant some skepticism, since in presented micrographs there are too many $\mathrm{GFP}^{+}$(GABAergic) cells, the soma of several neurons is pyramidal-like, and finally the size of GFP- (glutamatergic, if those black spots are intact neurons, but not damaged ones) cells are comparable to those of $\mathrm{GFP}^{+}$. Even during targeted recordings one would not encounter that amount of GABAergic cells in such a close proximity within the amygdala. This study is actually contrary to current dogma, since principal cells "exclusively showed regular spiking" and only about half of studied cells possess pyramidal soma. Moreover, the type-A GABAergic cells "spiked with little adaptation" and the size of their soma ranged up to $20 \mu \mathrm{m}$. 


\subsubsection{Plasticity in the amygdala and the role of $N E$}

NE release can be also triggered in brain slices by excessive depolarization using higher concentrations of $\mathrm{K}^{+}$. This procedure enables an increase (by 10-15\%) in NE content compared to basal magnitude in the amygdala (Lonart et al., 2008). Depolarization-induced NE release within the three nuclei were comparable in WT mice. In Rab3 interacting molecule 1 alpha $(\operatorname{Rim} 1 \alpha) \mathrm{KO}$ animals the identical approach evoked considerably lower NE release in BLA. Although in one of presented experiments, NE release from the CeA may have decreased, but the average data was not significant. The magnitude and response pattern of NE release in LA were indistinguishable from those of WT mice. Interestingly, in all these cases the peak release appeared with identical latencies of six minutes.

In control mice, NE gradually increases the frequency of spontaneous inhibitory postsynaptic currents (sIPSC) in pyramidal neurons of the BLA according to applied concentration (Braga et al., 2004). The effects of $1 \mu \mathrm{M} \mathrm{NE}$ was moderate, while of $10 \mu \mathrm{M}$ high and at $100 \mu \mathrm{M}$ excessive. Interestingly, at all tested doses the effects were completely reversible. The effects were observed also in the presence of adrenoceptor antagonists propranolol, yohimbine ( $\alpha 2 \mathrm{~A}), \mathrm{CEC}(\alpha 1 \mathrm{~B})$, and BMY $7378(\alpha 1 \mathrm{D})$. The selective $\alpha 1 \mathrm{~A}$ agonist A61603 increased both the rate and amplitude of sIPSC, and these effects were not observed in the presence of selective antagonist WB4101. NE at $10 \mu \mathrm{M}$ had opposite effects on evoked IPSC, and note that the inhibition was not complete, but reversible and perhaps rather targeted only to those with the highest amplitude. The results became more complicated, since a blockade of GABAв receptors (although their activation was not experimentally shown) changed the effects of NE on evoked IPSC. In the stressed (immobilized and exposed to tail-shocks) group of animals, the mean frequency of sIPSC slightly decreased ( 3.1 vs $2.6 \mathrm{~Hz}$ ). However, because of large diapason of values in individual experiments, the effects can not be considered significant. In these rats $10 \mu \mathrm{M}$ NE was ineffective.

Contrary to data of Braga et al. (2004), NE decreased the frequency of sIPSCs in pyramidal cells of LA, but did not alter their amplitude or any parameters of miniature events and eIPSCs (Tully et al., 2007). Therefore, it was assumed that NE decreases the excitability of interneurons. However, this assumption could be evidenced by recording APs and acutely applying NE. In some of the latter neurons, NE slightly decreased the tonic GABAergic currents, while the parameters of phasic ones were not altered. The conclusion of this study needs to be defined, since indeed the release of GABA into the pyramidal neurons was affected as it is supported by selective decrease of the frequency of sIPSCs by NE.

\subsubsection{Amygdala, placticity and fear learning}

An increase in synaptic strength within the amygdalar fear circuit comprises the plasticity. Among multiple amygdaloidal nuclei, the LA has been extensively studied. The LA is modulated by excitatory afferents and involved in synaptic plasticity, i.e. the counterpart that underlies learning and memory at neuronal level. Memory formation in the LA can easily be demonstrated, since synapses can undergo LTP (Fig. 3). 


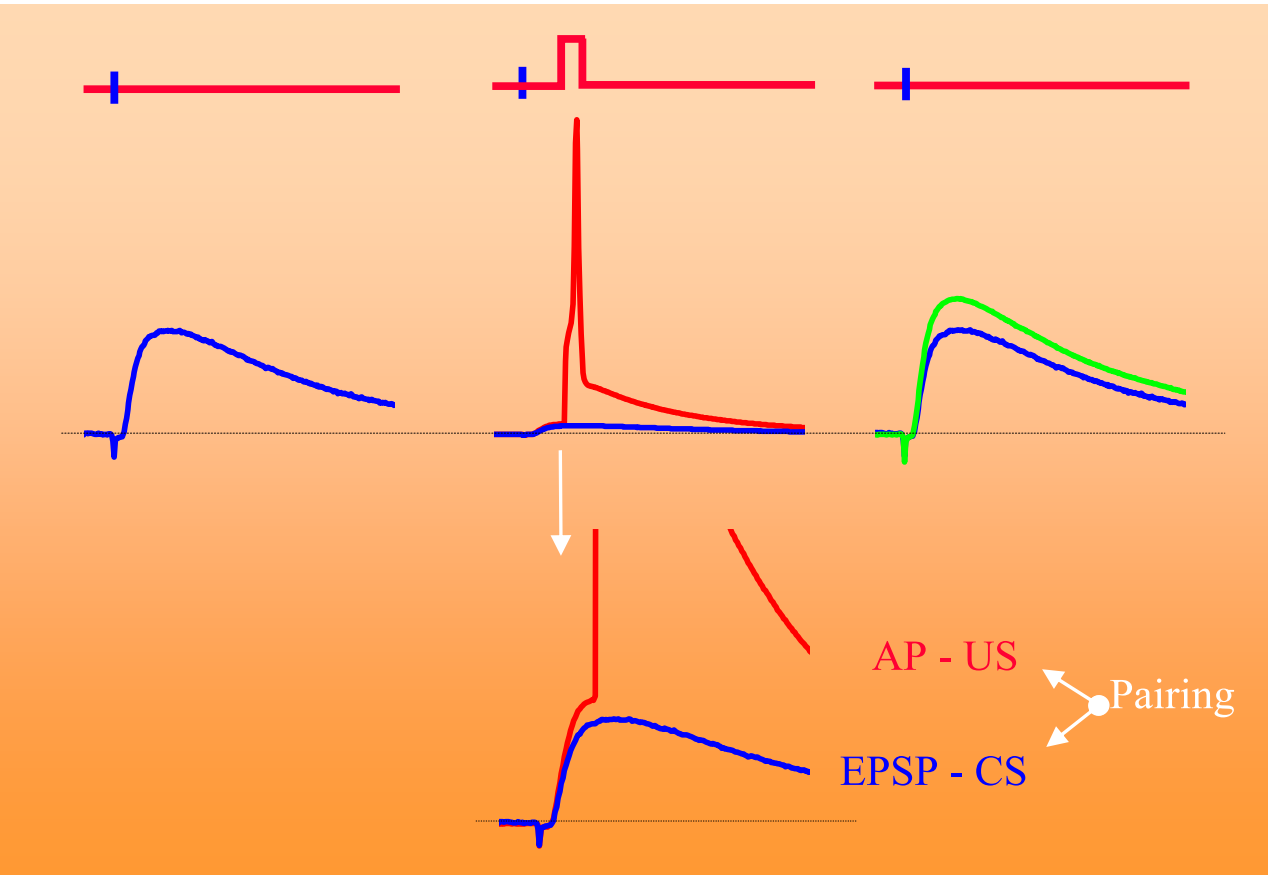

Figure 3. Induction of LTP in the LA in vitro

Mean baseline EPSP (blue trace) obtained in the presence of $100 \mu \mathrm{M}$ PTX by stimulation of cortical inputs into the pyramidal neuron. Pairing of EPSPs with depolarization-induced action potentials (red) at $2 \mathrm{~Hz}$ led to LTP (green).

LTP has been extensively explored and its properties in the amygdala are well established (Kodirov et al., 2006; Rogan et al., 1997; Royer \& Pare, 2003). In the amygdala, LTP induction can be either N-methyl-D-aspartate (NMDA) glutamate receptor dependent or independent (Mahanty \& Sah, 1998; Gewirtz \& Davis, 1997). Picrotoxin (PTX) facilitates the induction of LTP of excitatory postsynaptic potentials (EPSP) in the amygdala (Kodirov et al., 2006) by inhibiting the GABAA receptors. However, via the same mechanism, it can block the induction of another form of plasticity (Fink \& O'Dell, 2009) known as EPSP-spike (E-S) potentiation in cornu ammonis 1 (CA1). Under both of these circumstances, PTX decreases the level of inhibition of the principal cells during high frequency synaptic activation. The LTP time course can be subdivided into different components and subsequent early and late phases can be distinguished (Dong et al., 2008). The early phase of LTP (E-LTP) in vitro corresponds to short-term memory and the late phase (L-LTP) to long-term memory in vivo. The impairment in L-LTP correlates with the deficit in spatial and long-term memories (Abel et al., 1997).

Thus, despite some skepticism, the best-known counterpart of learning and memory at synaptic and input levels is LTP. LTP in the amygdala occurs at both the thalamic and cortical synapses into the pyramidal neurons. Recently, the NE effects on LTP at thalamic 
inputs into the LA were revealed (Tully et al., 2007). Interestingly, the latter effects were seen only under physiological conditions, i.e. under undisturbed balance of excitation and inhibition. Note that under these conditions, the synapses at thalamic inputs into the LA are not prone to LTP. Therefore, NE rather enables the induction of LTP, and its effects on maintenance remains to be elucidated. Under facilitated excitatory drive, which is commonly achieved in the presence of up to $100 \mu \mathrm{M}$ PTX, there was a tendency for enhanced LTP by NE, however, not to a significant level. Furthermore, the amplitudes of both components of glutamatergic currents remain unaltered in the presence of NE. However, two opposite effects were observed when prior to NE either $\alpha 2$ - (yohimbine) or $\beta$-adrenoreceptors (propranolol) antagonists were applied. During the priming with yohimbine NE increased the amplitude of evoked excitatory postsynaptic currents (EPSC), while with propranolol NE decreased it. The latency was shorter and magnitude was greater in the former situation compared to the second one. Both effects were to some extent reversible after application of either yohimbine or propranolol. It has previously been shown that the NE effects vary depending on targeted influence on the latter two receptors (Ferry et al., 1999a). Therefore, perhaps just by using either yohimbine or propranolol one could observe the corresponding drug induced LTP in those experiments in the LA.

NE increased the number of evoked action potentials in pyramidal neurons of the LA. Intrinsic excitability of neurons can be attributed per se to learning and memory in vivo and either the ability or disability of neurons to regulate this activity results in either memory formation or its impairment (Kaczorowski \& Disterhoft, 2009). The facilitated excitability of neurons by NE may contribute to the induction of LTP (if it occurs at least in part via inhibition of GABAergic or potentiation of glutamatergic neurotransmission, which can lead to increased postsynaptic depolarization during pairing with the presynaptic stimulation (Bissiere et al., 2003).

At thalamic inputs, terazosin decreased the amplitude of disynaptically induced inhibitory currents at $10 \mu \mathrm{M}$ concentration, which did not alter the excitatory ones that were evoked in response to the first pulse (Lazzaro et al., 2010). The sequence of dysynaptic excitatory and inhibitory currents/potentials can be also evoked by stimulating the cortical inputs and adjusting the holding potential to between -30 and $-40 \mathrm{mV}$ (Kodirov et al., 2006). Interestingly, the amplitude of excitatory currents evoked during application of second pulse was increased by terazosin (Lazzaro et al., 2010), thus resulting in paired-pulse facilitation (PPF). Note that no PPF at both synapses was observed under control conditions, although employing the ISI of $50 \mathrm{~ms}$ usually results in PPF when the single pulses are applied. Interestingly, the thalamo-amygdalar synapses exhibit LTP that decay with time, but in the presence of PTX it persisted steadily at least up to $1.5 \mathrm{~h}$. However, it is not clear when terazosin was applied, since the descriptions in both text and figures are contradictory. One pitfall during LTP experiments is the difficulty of comparing the potency of compounds, including NE. Nevertheless, at these inputs the LTP was more pronounced in the presence of terazosin compared to PTX. Since this compound was less effective at cortical synapses the authors conclude that the thalamic inputs are important in anxiety and $\alpha 1$ adrenoceptors play a role mostly in this pathway. 


\subsubsection{Molecular substrate of LTP}

The Pavlovian paradigm also alters the expression of multiple genes in amygdala. Under control conditions, freezing response was observed in $\sim 20 \%$, which was increased to $\sim 80 \%$ rats measured after $24 \mathrm{~h}$ of one trial of fear conditioning - pairing tone with foot-shock (Ploski et al., 2010). The behaviour of the rats was comparable in the next two trials. The qRT-PCR after $30 \mathrm{~min}$ of paradigm revealed an increase in mRNA of several genes: Arc-Activity-regulated cytoskeleton-associated protein, Egr2-Early growth response 2, Nr4a2-Nuclear receptor subfamily 4, group A, member 2, Per1-Period homolog 1 (Drosophila), Sat1-Spermidine/spermine Nl-acetyl transferase 1, Rnf39-Ring finger protein 39 increased. The expresion of Arc, Nr4a2, Per1 genes decreased by 90 min while those of remaining three increased. Also at $180 \mathrm{~min}$ two different responses were revealed. Among additional genes the highest increase at $30 \mathrm{~min}$ time point was revealed for Fos, although the degree of associated change is unclear ( 3 fold in the graph vs. 7 fold in the table). The latter perhaps is not a reflection of data normalizations, since the magnitude of corresponding genes in the naïve group was considered either as 0 or 1 , respectively. There are more molecular substrates potentially involved in LTP, and the active-zone scaffolding protein (RIM1 $\alpha$ ) is one of them (Fourcaudot et al., 2008).

We have encountered that the PPF at cortical inputs into the LA occurs less compared to other regions of the brain (Kodirov et al., 2009). A recent study demonstrates that the magnitude of PPF at these synapses decreases after a pairing paradigm from the ratio of $\sim 2$ to 1.5, at least one can estimate from representative traces (Fourcaudot et al., 2008). However, the average data is perhaps inadequately presented by subtracting the PPF of baseline from that of heterosynaptic associative LTP (LTPHA - evoked by the simultaneous stimulation of cortical and thalamic inputs), thus resulting in negative paired-pulse ratios (PPR). Comparing PPF before and after the paradigm would be sufficient in order to conclude whether or not the expression of LTP is presynaptic. Moreover, it is not clear that "changes in PPR could also involve postsynaptic mechanisms" and "induction of LTPнA depends on presynaptic, but not postsynaptic, NMDA receptors". The PPF ratio was reduced by $50 \mu \mathrm{M}$ forskolin and the identical concentration rapidly ( $2 \mathrm{~min}$ ) increases the EPSP reaching within four minutes the plateau and thus induces potentiation lasting at least 20 min. Its amplitude ( $150 \%$ of baseline) was comparable with LTP triggered by the stimulation of cortical inputs. Thus, PKA and RIM1 $\alpha$ were shown to alter the LTPHA.

\section{Conclusion}

NE influences memory performance either via interplay between the $\alpha$ - and $\beta$-adrenergic receptors or by co-activation of both (Ferry et al., 1999a, b). The memory promoting effects of NE via amygdala may occur also by the activation of principal neurons in the NTS and involve epinephrine (Williams et al., 1998). Amygdala dependent fear learning involves NE as a main neurotransmitter, since the post-training intracranial injection of $1 \mu \mathrm{g}$ NE led to amnesia in rats $24 \mathrm{~h}$ after passive avoidance task (Kesner \& Ellis, 1983). The pathological release of catecholamine NE in mammals including humans occurs in comparable manner to invertebrates (Kodirov, 2011). 
The above outlined dual effects of NE on memory (impairment and enhancement) possibly underly the distinct BLA single neuronal response, since iontophoretic injection of NE in some cells increased and others decreased the spontaneous firings in vivo (Buffalari \& Grace, 2007). These neurons exhibit different basal frequencies of firings, $\sim 0.1 \mathrm{vs.} 2 \mathrm{~Hz}$ in former and later, respectively. The fact that these neurons could be subdivided clearly into two groups is valuable, however identifying cells with higher rate as projecting ones (even based on their antidromic response to the stimulation of entorhinal cortex), is against the properties of neurons. The projection cells should not fire high frequency APs compared to other neurons within this structure, e.g. GABAergic interneurons. Nevertheless, the experiments are precise, since the recording and $200 \mu \mathrm{M}$ NE application was performed via the single multi-barrelled electrode at the same time. In the LA the proportion of neurons with inhibitory response to NE was higher than in the BLA, but those with excitatory ones similar. Note that NE did not alter firing of some neurons in either nuclei. Upon the termination of injection, the neurons continued to fire at baseline frequencies, therefore in the same neuron it was revealed that the NE inhibitory effects occur via $\alpha 2 \mathrm{AR}$, since the latter was mimicked by $50 \mu \mathrm{M}$ clonidine. The excitatory effects of NE on BLA neurons may occur via $\beta$ AR (for discussions see Buffalari \& Grace, 2007). Similar dual effects were observed also upon the stimulation of LC, but the baseline frequencies of two groups were only slightly apart. Although the extent of inhibition was similar after foot-shock or NE injection, the excitation was greater in the former case. The effects of NE were abrupt and those of foot-shock and LC stimulations occurred with adequate latencies.

Finally, the fear conditioning alone leads to the upregulation of $\sim 30$ genes in the LA (Ploski et al., 2010). Note that no downregulation for any genes was estimated in this study. Eventually, such results in the future could specify the role of plasticity related genes more precisely (in terms of their associations to certain neurotransmitters including NE), which then potentially could serve as a target points during diagnosis and the search for potential cure. Even in this century "how memory processing would be coded at the receptor [or gene] level remains unknown" (Ellis, 1985).

\section{Author details}

\section{Sodikdjon A. Kodirov}

Pavlov Institute of Physiology, Russian Academy of Sciences, Saint Petersburg, Russia

I. P. Pavlov Department of Physiology, State Research Institute of Experimental Medicine, Russian

Academy of Medical Sciences, Saint Petersburg, Russia

Department of Molecular Physiology \& Biophysics, University of Iowa, Iowa City, USA

\section{Acknowledgement}

In this instance, I would like to acknowledge contributions of authors, which I was not able to review because of either length limitations or many of them were discussed elsewhere recently. I am also grateful to Nicholas D. Leymaster and Carl J. Christel for reading this work at its final stage. 


\section{References}

Abel T, Nguyen PV, Barad M, Deuel TAS, Kandel ER \& Bourtchouladze R. 1997. Genetic demonstration of a role for PKA in the late phase of LTP and in hippocampus-based long-term memory. Cell 88:615-626.

Akirav I, Richter-Levin G. 2002. Mechanisms of amygdala modulation of hippocampal plasticity. J Neurosci 22:9912-21

Alberini CM. 2008. The role of protein synthesis during the labile phases of memory: Revisiting the skepticism. Neurobiol Learn Mem 89:234-246.

Almaguer-Melian W, Cruz-Aguado R, Riva Cde L, Kendrick KM, Frey JU, Bergado J. 2005. Effect of LTP-reinforcing paradigms on neurotransmitter release in the dentate gyrus of young and aged rats. Biochem Biophys Res Commun 327:877-83

Arnsten AF. 2007. Catecholamine and second messenger influences on prefrontal cortical networks of "representational knowledge": a rational bridge between genetics and the symptoms of mental illness. Cereb Cortex 17 Suppl 1:i6-15

Bergado JA, Frey S, Lopez J, Almaguer-Melian W, Frey JU. 2007. Cholinergic afferents to the locus coeruleus and noradrenergic afferents to the medial septum mediate LTPreinforcement in the dentate gyrus by stimulation of the amygdala. Neurobiol Learn Mem 88:331-41

Berlau DJ, McGaugh JL. 2006. Enhancement of extinction memory consolidation: the role of the noradrenergic and GABAergic systems within the basolateral amygdala. Neurobiol Learn Mem 86:123-32

Bissiere S, Humeau Y \& Luthi A. 2003. Dopamine gates LTP induction in lateral amygdala by suppressing feedforward inhibition. Nat Neurosci 6, 587-592.

Braga MF, Aroniadou-Anderjaska V, Manion ST, Hough CJ, Li H. 2004. Stress impairs $\alpha_{1 A}$ adrenoceptor-mediated noradrenergic facilitation of GABAergic transmission in the basolateral amygdala. Neuropsychopharmacology 29:45-58

Brager DH \& Johnston D. 2007. Plasticity of intrinsic excitability during long-term depression is mediated through mGluR-dependent changes in Ih in hippocampal CA1 pyramidal neurons. J Neurosci 27:13926-13937

Bremner JD. 2006. Traumatic stress: effects on the brain. Dialogues Clin Neurosci 8:445-61

Brodal A. 1947. The amygdaloid nucleus in the rat. J Comp Neurol 87:1-16

Buffalari DM, Grace AA. 2007. Noradrenergic modulation of basolateral amygdala neuronal activity: opposing influences of $\alpha-2$ and $\beta$ receptor activation. J Neurosci 27:12358-66

Cahill L, Gorski L \& Le K. 2003. Enhanced human memory consolidation with post-learning stress: Interaction with the degree of arousal at encoding. Learn. Mem. 10: 270-274

Canal CE, Chang Q, Gold PE. 2007. Amnesia produced by altered release of neurotransmitters after intraamygdala injections of a protein synthesis inhibitor. Proc Natl Acad Sci U S A 104:12500-5

Canal CE, Chang Q, Gold PE. 2008. Intra-amygdala injections of CREB antisense impair inhibitory avoidance memory: role of norepinephrine and acetylcholine. Learn Mem 15:677-86 
Cowan WM, Raisman G, Powell TP. 1965. The connexions of the amygdala. J Neurol Neurosurg Psychiatry 28:137-51

Davies MF, Tsui J, Flannery JA, Li X, DeLorey TM, Hoffman BB. 2004. Activation of alpha2 adrenergic receptors suppresses fear conditioning: expression of c-Fos and phosphorylated CREB in mouse amygdala. Neuropsychopharmacology 29:229-39

Dong C, Upadhya SC, Ding L, Smith TK \& Hegde AN. 2008. Proteasome inhibition enhances the induction and impairs the maintenance of late-phase long-term potentiation. Learn Mem 15, 335-347.

Ellis ME. 1985. Amygdala norepinephrine involved in two separate long-term memory retrieval processes. Brain Res 342:191-5

Emson PC, Björklund A, Lindvall O, Paxinos G. 1979. Contributions of different afferent pathways to the catecholamine and 5-hydroxytryptamine-innervation of the amygdala: A neurochemical and histochemical study. Neuroscience 4:1347-57

Ferry B, McGaugh JL. 2008. Involvement of basolateral amygdala $\alpha 2$-adrenoceptors in modulating consolidation of inhibitory avoidance memory. Learn Mem 15:238-43

Ferry B, Roozendaal B, McGaugh JL. 1999a. Basolateral amygdala noradrenergic influences on memory storage are mediated by an interaction between $\beta$ - and $\alpha 1$-adrenoceptors. J Neurosci 19:5119-23

Ferry B, Roozendaal B, McGaugh JL. 1999b. Involvement of $\alpha 1$-adrenoceptors in the basolateral amygdala in modulation of memory storage. Eur J Pharmacol 372:9-16

Fink AE \& O'Dell TJ. 2009. Short trains of theta frequency stimulation enhance CA1 pyramidal neuron excitability in the absence of synaptic potentiation. J Neurosci 29, 11203-11214.

Fourcaudot E, Gambino F, Humeau Y, Casassus G, Shaban H, Poulain B, Lüthi A. 2008. cAMP/PKA signaling and RIM1 $\alpha$ mediate presynaptic LTP in the lateral amygdala Proc Natl Acad Sci U S A 105:15130-15135

Gewirtz JC \& Davis M. 1997. Second-order fear conditioning prevented by blocking NMDA receptors in amygdala. Nature 388, 471-474.

Gidron Y, Barak T, Henik A, Gurman G, Stiener O. 2002. Implicit learning of emotional information under anesthesia. Neuroreport 13:139-42

Groch S, Wilhelm I, Diekelmann S, Sayk F, Gais S, Born J. 2011. Contribution of norepinephrine to emotional memory consolidation during sleep. Psychoneuroendocrinology 36:1342-50

Gurdjian ES. 1928. The corpus striatum of the rat. Studies on the brain of the rat. No. 3. J Comp Neurol 45:249-81

Halverson HE, Hubbard EM \& Freeman JH. 2009. Stimulation of the lateral geniculate, superior colliculus, or visual cortex is sufficient for eyeblink conditioning in rats. Learn Mem 16, 300-307.

Huff NC, Wright-Hardesty KJ, Higgins EA, Matus-Amat P, Rudy JW. 2005. Context preexposure obscures amygdala modulation of contextual-fear conditioning. Learn Mem 12:456-60

Hurlemann R, Hawellek B, Matusch A, Kolsch H, Wollersen H, et al. 2005. Noradrenergic modulation of emotion-induced forgetting and remembering. J Neurosci 25:6343-9 
Hurlemann R, Matusch A, Hawellek B, Klingmuller D, Kolsch H, et al. 2007. Emotioninduced retrograde amnesia varies as a function of noradrenergic-glucocorticoid activity. Psychopharmacology (Berl) 194:261-9

Kaczorowski CC \& Disterhoft JF. 2009. Memory deficits are associated with impaired ability to modulate neuronal excitability in middle-aged mice. Learn Mem 16, 362-366.

Kaneko K, Tamamaki N, Owada H, Kakizaki T, Kume N, et al. 2008. Noradrenergic excitation of a subpopulation of GABAergic cells in the basolateral amygdala via both activation of nonselective cationic conductance and suppression of resting $\mathrm{K}^{+}$ conductance: a study using glutamate decarboxylase 67-green fluorescent protein knock-in mice. Neuroscience 157:781-97

Kesner RP, Ellis ME. 1983. Memory consolidation: brain region and neurotransmitter specificity. Neurosci Lett 39:295-300

Knierim JJ. 2003. Hippocampus and memory. Can we have our place and fear it too? Neuron 37, 372-374.

Kodirov SA. 2011. The neuronal control of cardiac functions in Molluscs. Comp Biochem Physiol A. 160: 102-116.

Kodirov SA, Jasiewicz J, Amirmahani P, Psyrakis D, Bonni K, et al. 2009. Endogenous cannabinoids trigger the depolarization-induced suppression of excitation in the lateral amygdala. Learn Mem 17:43-9

Kodirov SA, Takizawa S, Joseph J, Kandel ER, Shumyatsky GP, Bolshakov VY. 2006. Synaptically released zinc gates long-term potentiation in fear conditioning pathways. Proc Natl Acad Sci U S A 103:15218-23

Kwapis JL, Jarome TJ, Lonergan ME \& Helmstetter FJ. 2009. Protein kinase Mzeta maintains fear memory in the amygdala but not in the hippocampus. Behav Neurosci 123, 844-850.

LaLumiere RT, Buen TV, McGaugh JL. 2003. Post-training intra-basolateral amygdala infusions of norepinephrine enhance consolidation of memory for contextual fear conditioning. J Neurosci 23:6754-8

Lazzaro SC, Hou M, Cunha C, LeDoux JE, Cain CK. 2010. Antagonism of lateral amygdala alpha1-adrenergic receptors facilitates fear conditioning and long-term potentiation. Learn Mem 17:489-93

LeDoux JE, Sakaguchi A, Reis DJ. 1984. Subcortical efferent projections of the medial geniculate nucleus mediate emotional responses conditioned to acoustic stimuli. J Neurosci 4:683-98.

Li R, Nishijo H, Wang Q, Uwano T, Tamura R, et al. 2001. Light and electron microscopic study of cholinergic and noradrenergic elements in the basolateral nucleus of the rat amygdala: evidence for interactions between the two systems. J Comp Neurol 439:41125

Lonart G, Tang X, Simsek-Duran F, Machida M, Sanford LD. 2008. The role of active zone protein Rab3 interacting molecule 1 alpha in the regulation of norepinephrine release, response to novelty, and sleep. Neuroscience 154:821-31

Mahanty NK \& Sah P. 1998. Calcium-permeable AMPA receptors mediate long-term potentiation in interneurons in the amygdala. Nature 394, 683-687. 
McIntyre CK, Hatfield T, McGaugh JL. 2002. Amygdala norepinephrine levels after training predict inhibitory avoidance retention performance in rats. Eur J Neurosci 16:1223-6

McKernan MG \& Shinnick-Gallagher P. 1997. Fear conditioning induces a lasting potentiation of synaptic currents in vitro. Nature 390, 607-611.

McReynolds JR, Donowho K, Abdi A, McGaugh JL, Roozendaal B, McIntyre CK. 2010. Memory-enhancing corticosterone treatment increases amygdala norepinephrine and Arc protein expression in hippocampal synaptic fractions. Neurobiol Learn Mem 93:312-21

Mikula, S., Trotts, I., Stone, J., and Jones, E.G. 2007. Internet-enabled high-resolution brain mapping and virtual microscopy. NeuroImage. 35:9-15

Murchison CF, Schutsky K, Jin SH, Thomas SA. 2011. Norepinephrine and $\beta 1$-adrenergic signaling facilitate activation of hippocampal CA1 pyramidal neurons during contextual memory retrieval. Neuroscience 181:109-16

Niwa M, Matsumoto Y, Mouri A, Ozaki N, Nabeshima T. 2011. Vulnerability in early life to changes in the rearing environment plays a crucial role in the aetiopathology of psychiatric disorders. Int J Neuropsychopharmacol 14:459-77

Onaka T, Yagi K. 1998. Role of noradrenergic projections to the bed nucleus of the stria terminalis in neuroendocrine and behavioral responses to fear-related stimuli in rats. Brain Res 788:287-93

Pavlov IP. 1927. Conditioned Reflexes. Dover, New York.

Paxinos G \& Franklin KBJ. 2001. The mouse brain in stereotaxic coordinates. Academic Press.

Peters J, Kalivas PW \& Quirk GJ. 2009. Extinction circuits for fear and addiction overlap in prefrontal cortex. Learn Mem 16, 279-288.

Ploski JE, Pierre VJ, Smucny J, Park K, Monsey MS, et al. 2008. The activity-regulated cytoskeletal-associated protein (Arc/Arg3.1) is required for memory consolidation of Pavlovian fear conditioning in the lateral amygdala. J Neurosci 28, 12383-95

Poe GR, Nitz DA, McNaughton BL \& Barnes CA. 2000. Experience-dependent phasereversal of hippocampal neuron firing during REM sleep. Brain Res 855, 176-180.

Radwanska K, Nikolaev E, Kaczmarek L. 2010. Central noradrenergic lesion induced by DSP-4 impairs the acquisition of avoidance reactions and prevents molecular changes in the amygdala. Neurobiol Learn Mem 94:303-11

Reijmers LG, Perkins BL, Matsuo N \& Mayford M. 2007. Localization of a stable neural correlate of associative memory. Science 317, 1230-1233.

Rogan MT, Staubli UV \& LeDoux JE. 1997. Fear conditioning induces associative long-term potentiation in the amygdala. Nature 390, 604-607.

Roozendaal B, Hui GK, Hui IR, Berlau DJ, McGaugh JL, Weinberger NM. 2006a. Basolateral amygdala noradrenergic activity mediates corticosterone-induced enhancement of auditory fear conditioning. Neurobiol Learn Mem 86:249-55

Roozendaal B, Okuda S, Van der Zee EA, McGaugh JL. 2006b. Glucocorticoid enhancement of memory requires arousal-induced noradrenergic activation in the basolateral amygdala. Proc Natl Acad Sci U S A 103:6741-6 
Roozendaal B, Castello NA, Vedana G, Barsegyan A, McGaugh JL. 2008. Noradrenergic activation of the basolateral amygdala modulates consolidation of object recognition memory. Neurobiol Learn Mem 90:576-9

Royer S \& Pare D. 2003. Conservation of total synaptic weight through balanced synaptic depression and potentiation. Nature 422, 518-522.

Sadowski RN, Canal CE, Gold PE. 2011. Lidocaine attenuates anisomycin-induced amnesia and release of norepinephrine in the amygdala. Neurobiol Learn Mem 96:136-42

Smolka MN, Schumann G, Wrase J, Grusser SM, Flor H, et al. 2005. Catechol-Omethyltransferase val158met genotype affects processing of emotional stimuli in the amygdala and prefrontal cortex. J Neurosci 25:836-42

Tully K, Li Y, Tsvetkov E, Bolshakov VY. 2007. Norepinephrine enables the induction of associative long-term potentiation at thalamo-amygdala synapses. Proc Natl Acad Sci U S A 104:14146-50

Varshney LR, Sjostrom PJ \& Chklovskii DB. 2006. Optimal information storage in noisy synapses under resource constraints. Neuron 52, 409-423.

van Stegeren AH, Goekoop R, Everaerd W, Scheltens P, Barkhof F, et al. 2005. Noradrenaline mediates amygdala activation in men and women during encoding of emotional material. Neuroimage 24:898-909

van Stegeren AH, Roozendaal B, Kindt M, Wolf OT, Joels M. 2010. Interacting noradrenergic and corticosteroid systems shift human brain activation patterns during encoding. Neurobiol Learn Mem 93:56-65

van Stegeren AH, Wolf OT, Everaerd W, Scheltens P, Barkhof F, Rombouts SA. 2007. Endogenous cortisol level interacts with noradrenergic activation in the human amygdala. Neurobiol Learn Mem 87:57-66

Wagner U, Degirmenci M, Drosopoulos S, Perras B, Born J. 2005. Effects of cortisol suppression on sleep-associated consolidation of neutral and emotional memory. Biol Psychiatry 58:885-93

Williams CL, Men D, Clayton EC, Gold PE. 1998. Norepinephrine release in the amygdala after systemic injection of epinephrine or escapable footshock: contribution of the nucleus of the solitary tract. Behav Neurosci 112:1414-22 